\title{
Customized Industrial Networks - Network Slicing Trial at Hamburg Seaport
}

\author{
Peter Rost ${ }^{1}$, Markus Breitbach ${ }^{2}$, Hendrik Roreger ${ }^{3}$, \\ Bilgehan Erman ${ }^{4}$, Christian Mannweiler ${ }^{1}$, Ray Miller ${ }^{4}$, Ingo Viering ${ }^{5}$ \\ ${ }^{1}$ Nokia Bell Labs, Munich, Germany \\ ${ }^{2}$ Deutsche Telekom, Bonn, Germany \\ ${ }^{3}$ Hamburg Port Authority, Hamburg, Germany \\ ${ }^{4}$ Nokia Bell Labs, Murray Hill, USA \\ ${ }^{5}$ Nomor Research GmbH, Munich, Germany
}

\begin{abstract}
Driven by a massive surge in digitization and customization, so-called vertical industries are expected to be a major beneficiary of the fifth generation $(5 \mathrm{G})$ of mobile networks. The use cases of such vertical industries define qualitative and quantitative requirements unprecedented in the history of mobile network development. Autonomous vehicles, traffic light control, video surveillance, industrial Internet of Things (IIoT), to only name a few, introduce challenging requirements regarding both conventional performance metrics, such as, throughput or coverage, as well as formerly rather subordinate system metrics, such as deterministic latency, ultra-high reliability and resilience, high number of devices, multi-tenant networks, or demanding security mechanisms.

Nokia, Deutsche Telekom, and Hamburg Port Authority have deployed a large-scale 5G trial testbed in the Hamburg port area. The testbed proves in a real, large-scale industrial environment that basic features of network slicing, namely slice isolation, flexible slice customization and multi-tenancy, are technically feasible already today. Three exemplary communication services have been selected and are demonstrated in the testbed. Multi-connectivity is implemented as a key component to achieve high reliability throughout the testbed area. The testbed shows that all network domains must be involved in the setup of network slices, i.e., user terminals, radio access, core network, and enterprise networks, in order to efficiently operate and manage network slices. Therefore, the discussed Life Cycle Management is key for the interaction between mobile service provider and tenants of the network.
\end{abstract}

Index Terms-5G Trial, Hamburg seaport, Network Slicing, Life Cycle Management, Multi-Connectivity

\section{INTRODUCTION}

\section{A. Digital Industrial Network - A Guarantee for Economic Growth}

The $5^{\text {th }}$ generation of mobile communications facilitates a trend of fundamental change towards digitization in industries. One such example of a vertical industry are seaports. Seaports have always reflected changes in global economy. Sixty years ago, the introduction of containers revolutionized maritime logistics and seaports quickly adapted to this challenge. Digitalization is the disruptive challenge in maritime industries these days. Long established global value chains are heavily affected by this megatrend. Technologies like 3Dprinting, robotics, machine learning, virtual reality, or the Internet of Things (IoT) change production processes in many industries. Customers adapt to these possibilities and demand a broader range of products, which are heavily customized to each individual need. Therefore, manufacturers need to produce manifold product variants. Still, businesses and consumers are used to receiving goods quickly and at a planned date, e.g., just-in-time delivery for production lines. Manufacturers need to be able to control and optimize the whole production process from order to delivery while still maintaining the immense variety of products in the era of digitization.

The centerpiece of the global supply chain is maritime logistics. Its value chains consist of maritime transport, hinterland transport, infrastructure, and business models. It is a value creation network, where shipping lines 
are the edges connecting seaports [1]. Shipping time is defined by oversea travel times, which has already been optimized between environmental and economic factors. That brings maritime logistics focus on optimization to seaports, which are the crucial link between shipping and hinterland transport. Processes in seaports have evolved at a slow rate for centuries, but technological innovations will have enormous potential in the manifold processes of seaports. Key challenge is digital logistics-traffic optimization in safe and secure manner while maintaining and even reducing environmental impacts of ports.

One of the most important areas of innovation is the Internet of Things. All assets, vehicles, containers and traffic lights will be represented by a digital twin, which allows for measuring data and controlling actuators, e.g., traffic lights and vehicles connected to a central infrastructure to enable real-time traffic management. Optimal traffic management is crucial for seaports, e.g., in Port of Hamburg up to 10,000 trucks traverse the port area each day. Congestions of trucks delay and therefore negatively impact the supply chain. Mobile communication in the whole supply chain will optimize processes, and gathering and analyzing data in realtime will implement a resilient process.

\section{B. $5 G-A$ Key to Digital Customization}

In order to enable industrial communication, $5 \mathrm{G}$ addresses use cases and requirements of vertical industries. Thus, 5G will support not only massive broadband (MBB) but also mission critical communication (cMTC), and massive machine communication (mMTC). The design of the mobile network needs to consider the following aspects:

- A diversity of network infrastructure and services needs to be provided within the area of the seaport, ranging from critical applications (e.g., control of water gates) to massive broadband applications (e.g., offering Internet to passengers of large cruise ships), or massive sensor applications.

- Failure of the Information and Communication Technology (ICT) infrastructure may have international impact, e.g., a stoppage at a central hub such as Hamburg may have an impact on goods transport in all central Europe.

- The ICT infrastructure may be subject to cyber-attacks on critical company data and critical infrastructure.

As a result, the main requirements of this vertical include resilience (guaranteed availability, even in the case of failures), dynamic instantiation and management of isolated logical networks (network slices), and support for service diversity. To demonstrate the ability of $5 \mathrm{G}$ to satisfy these requirements, we deploy a testbed implementing different use cases. It is located at the Hamburg seaport, and the testbed is operated by Deutsche Telekom (DT), Hamburg Port Authority (HPA), and Nokia.

In the next section, use cases and requirements are detailed; in Section III, the testbed setup is described in further detail; in Section IV, key technology implementations are outlined; and Section V concludes the paper.

\section{USE CASES AND REQUIREMENTS}

Currently, a multitude of applications is already operational in the Hamburg seaport, such as environmental monitoring, location information of ships, level metering, and traffic management. At present, a major part of these applications is implemented through (partly analogue) fixed line networks, which lack the scalability and flexibility required by future applications involving a substantially larger number of devices. The challenges involved range from pure infrastructure problems (i.e., connecting devices) to application-layer problems (i.e., interworking of protocols). This will be represented through the following three applications implemented in the testbed:

1. Traffic light control (cMTC): The testbed will connect traffic lights through wireless links, i.e., connections must be reliable and resilient; traffic lights may be added or removed over time; and security and data integrity are important. 
2. Live Remote Site Support (MBB): Live Video assistance or assistance by Virtual- and AugmentedReality is needed to support sites remotely, e.g. construction sites or traffic congestions; no side effects to cMTC are allowed; reliable connections are needed; and data privacy and security are important due to regulations.

3. Sensor measurements (mMTC): Sensor measurements on barges (small sized boats) must be connected through wireless terminals, which yields a scenario with many terminals with uplink traffic of varying amount and mobility requirements.

\section{A. Customization and Isolation}

The above listed applications reflect the highly heterogeneous requirements on the underlying communication network. The traditional "one size fits all" approach, i.e., one general purpose communications network serving all kinds of applications, will likely result in an unsatisfactory performance for the majority of customer applications. A near-at-hand solution might be building an own network for each application; however, this solution would be very inflexible and economically not viable in most cases.

The approach implemented in this testbed is an integral 5G feature: Network slicing [2][3], i.e., all applications share a common infrastructure, which hosts multiple logical networks. The logical networks may differ with respect to their topology, functionality and quality of the provided communication services, and they can be designed and configured individually to meet the needs of applications. Hence, 5G mobile network infrastructure will support customized self-contained logical network instances where the actual network functions for each slice may be independently operated and customized based on the service requirements.

A key enabler for this customization is the ability to flexibly decompose and allocate network functions. The location of network functions can be dependent on the service requirements such as on throughput, latency, but also security and privacy. For instance, highly critical services and networks may only use local resources and a dedicated high-security context while less critical services may use less stringent and resource-intense security implementations.

In addition to the service characteristics, also the infrastructure properties in the communication and industrial domain must be taken into account. Furthermore, the services need to be isolated to avoid side-effects on resource allocations as well as to ensure data integrity and privacy. For instance, depending on the service requirements, resources may be multiplexed or static resources may need to be allocated to meet stringent requirements on latency and throughput. Nevertheless, changing the resource allocation of one service must not lead to a violation of service level agreements (SLAs) of another service. In our testbed, this customization is provided by a network slicing implementation and a corresponding Life Cycle Management as detailed in Section IV.A.

\section{B. Multi-Tenancy}

In the Hamburg port setup, multiple stakeholders interact in order to realize the end-to-end communication service. Figure 1 depicts a generalization of the complex ecosystem of stakeholders [4].

The mobile service provider (MSP) depicts the business providing Internet connectivity and telecommunication services to subscribers - namely DT (in the context of this trial). The MSP offers dedicated mobile network instances (i.e., "Network Slice as a Service", NSaaS) that realize a specified telecommunication service, e.g., mMTC, to external and internal tenants, such as, HPA or internal departments. The tenant, usually a business entity, buys and leverages on the MSP's network slice services. Further, the infrastructure provider (InP) is the business that owns and manages parts or all of the infrastructure of the network. In the context of this trial, both DT and HPA have the role of an InP. Nokia has the role of the software and hardware vendor for the deployed mobile network. Finally, depending on the tenant, end devices can be very diverse. In the Hamburg port use cases, they include both IoT devices and mobile users operating in the port area. 


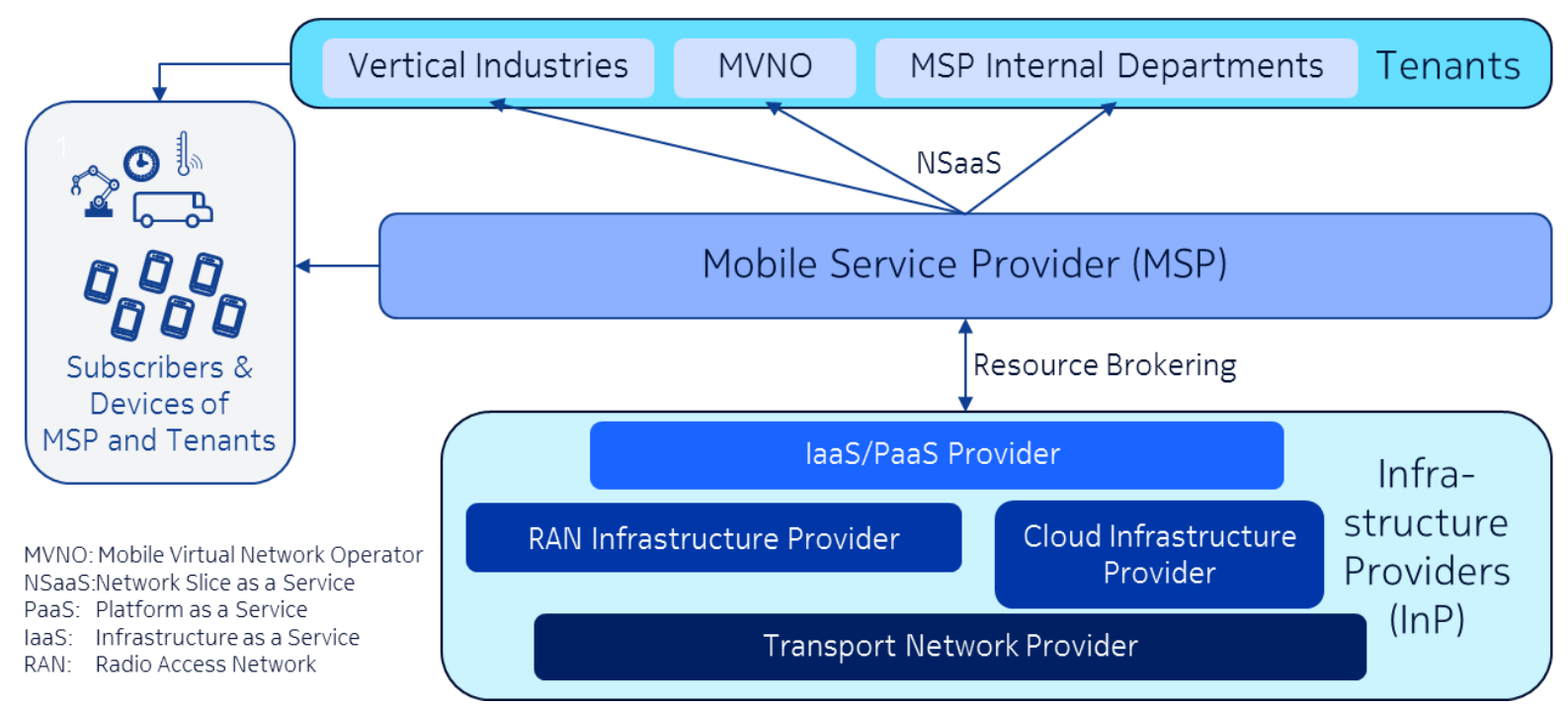

Figure 1: Relationship between stakeholders in the 5G ecosystem

In the described model, the goal of HPA (and potentially other tenants) is to provide services and applications to their end-user subscribers with contracted quality of experience, alongside the mobile communication service provided by the MSP (i.e., DT). Therefore, they have a commercial agreement with the MSP to use an end-to-end virtual network, i.e., a network slice. It usually is the role of the MSP (DT) to acquire the necessary resources from one or more InPs (here, DT and HPA) to build an end-to-end logical network (slice) instance according to the needs of the tenant. The MSP can either operate the logical network on behalf of the tenant or expose network configuration options to the tenant.

\section{Reliability}

In a seaport communication network, reliable and deterministic connectivity even in large coverage areas is very important. Typical applications in areas such as Hamburg are remote control of water gates, traffic lights, cranes, or smaller boats. In those cases, the control loop will require availability, reliability, and resiliency. Availability refers to the ability to connect to the network at any place within the coverage area; reliability refers to the quality of service guarantee once the connection has been established; and resiliency refers to ability of the network to quickly recover from failures in the infrastructure such as sudden interruption of a base station signal (e.g., blockage).

A key concept to achieve the reliability of the network is to provide multiple connectivity options such that the same user terminal would connect to different radio access points. This concept is implemented in the Hamburg testbed and further described in Section IV.B.

\section{Testbed Setup}

\section{A. Baseline assumptions}

The Hamburg port testbed trials network slicing in the context of industrial or commercial applications. It shows the feasibility of essential components of network slicing as described in [5] and demonstrates added values to industrial applications.

Commercially available equipment (particularly in radio access network (RAN)) is used as far as possible and the hardware is extended by features specifically needed to satisfy the requirements, detailed in Section II, and which would be available within the next two to three years. Where necessary, proprietary equipment is deployed. For demonstrating architectural aspects of network slicing and to verify that network slicing infrastructure could meet the requirements of the customers' applications, the radio technology of the air interface is of secondary importance. Hence, 3GPP LTE Rel. 14 [6] has been used and modified when nec- 
essary. In the testbed, a particular focus was on mechanisms allowing to ensure industry-grade network reliability. The detailed assessment of the outcome of the study is expected to show which improvements can be achieved by such mechanisms and how the infrastructure and other slices are impacted.

Currently, 3GPP LTE offers the opportunity of implementing dedicated logical core networks (CNs) for specific purposes using "eDECOR" [7]. While this "early form" of network slicing offers opportunities particularly for IoT deployments, it does not enable E2E Network Slicing including terminals, RAN, and the dynamic deployment of network slices (incl. multiple network slices per terminal). Standardization work on network slicing is ongoing in 3GPP Release 15 and will continue in Release 16. 3GPP Release 15 marks the completion of 5G Phase 1 including 5G New Radio and 5G Core, which will support E2E Network Slicing.

The number of slices that can be hosted by a common infrastructure shall be large. While the exact scale is still under discussion, it is clear that it will be too large for a purely manual operation. Hence, automated operational processes are required. Novel orchestration and management tools have to be developed that are not only capable of adjusting network parameters in a single network, but in addition can also tune parameter settings and inter-slice resource allocation to optimize overall resource efficiency.

An essential pre-requisite for inter-slice resource allocation is the understanding of indicators that appropriately reflect the performance of a network slice from the perspective of the customer's application, and how these indicators depend on slice configuration and resource allocation. Hence, the network operator would know the level of service reliability that can be offered to the tenant, and would allow the operator to manage the network in compliance with target SLAs. Indicators used as basis for SLA compliance between tenant and network operator should be verifiable equally well by both parties.

\section{B. A Network Slicing Reference Architecture}

Figure 2 depicts a network architecture designed to deliver heterogeneous communication profiles over a shared infrastructure [5]. It is separated into four layers, each of them realizing a defined subset of the operational tasks required in a productive communication system that is shared between a mobile network operator (DT) and a third-party tenant (HPA). This architecture is used as basis for the Hamburg testbed.

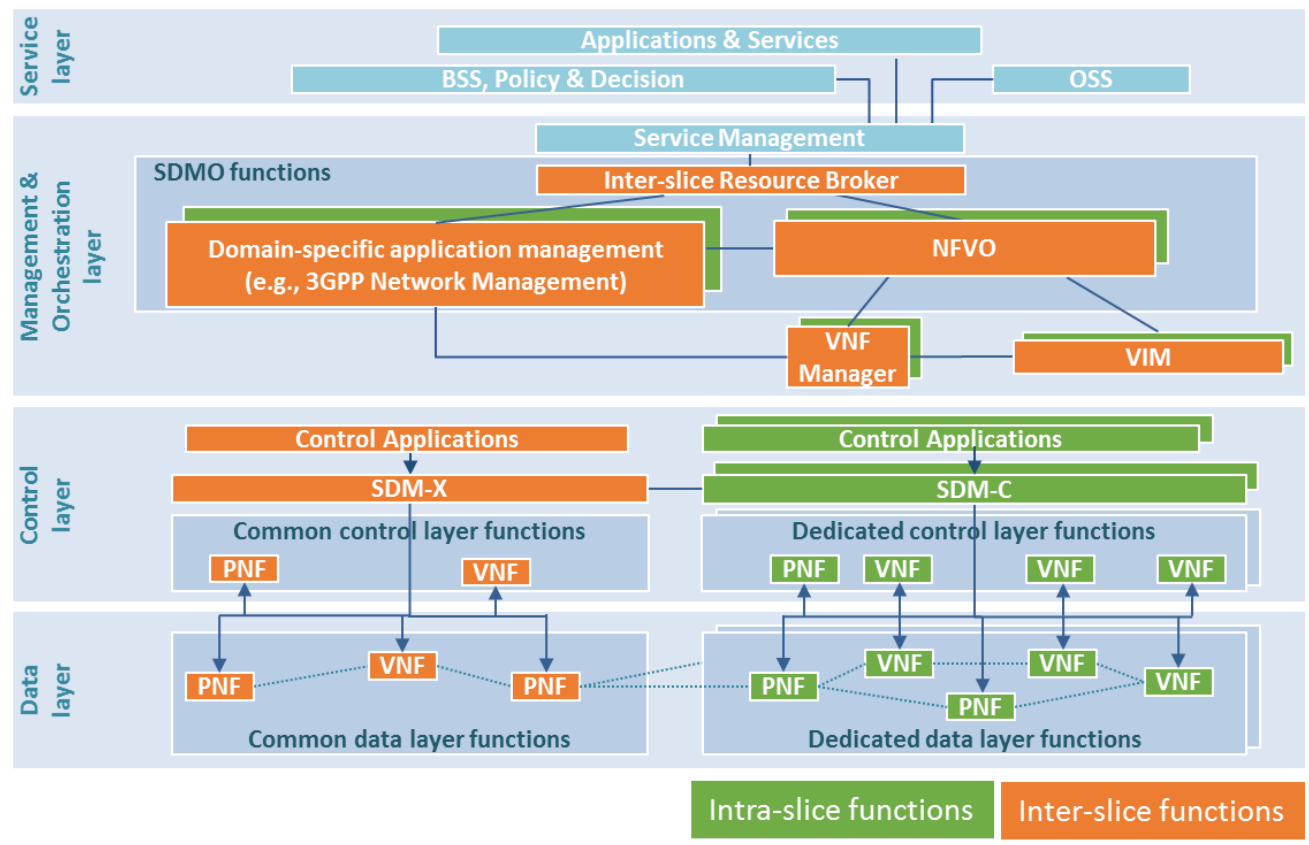

Figure 2: Functional perspective of the overall 5G NORMA architecture 
The Service Layer comprises Operations and Business Support Systems (OSS, BSS) including businesslevel Policy and Decision functions, as well as applications and services operated by the tenant (HPA), e.g., traffic light control, sensor data processing, or video surveillance analysis.

The Management \& Orchestration (M\&O) Layer realizes a Software-defined Management \& Orchestration (SDMO) concept by extending the ETSI Network Function Virtualization (NFV) management and orchestration (NFV MANO) architecture [8] towards multi-tenant and multi-service networks. The layer therefore comprises the functions required for lifecycle management (LCM) of virtualized network functions and resources. Further, the layer accommodates application management functions from various domains, e.g., for cellular network management provided by DT or enterprise network management by HPA. Moreover, a so-called Inter-Slice Resource Broker (ISRB) enforces policies for cross-slice resource allocation. Finally, the Service Management is an intermediary function between the Service layer and the ISRB. It transforms consumer-facing service descriptions provided by HPA entities into resource-facing service descriptions (particularly, target network performance). Further, it reports network performance to HPA Service layer functions and exposes limited network configuration APIs towards HPA.

The Control layer accommodates the two controllers: (1) the Software-Defined Mobile Network Coordinator (SDM-X), operated by DT for the control of common (shared) network functions (NFs), and (2) the Software-Defined Mobile Network Controller (SDM-C) for dedicated NFs of DT or HPA, respectively. Following the Software-Defined Networking principles, SDM-X and SDM-C abstract from the technological and implementation-related details of controlled NFs and facilitate NF programmability.

Finally, the Data layer comprises the virtual NFs (VNFs) and physical NFs (PNFs) needed to carry and process the user data traffic. A network slice, i.e., the set of Control and Data layer functions, can span multiple network domains, including (radio) access, core, transport, and private enterprise domains. Each of the three use cases described in Sec. II are implemented by a separate network slice instance.

This architecture is compliant to the network slicing approach defined in 3GPP. For the CN, [9] defines a network slice to include the CN Control Plane and User Plane NFs. In the context of Figure 2, these can be directly mapped to the intra- or inter-slice Control layer and Data layer NFs, respectively. The 3GPP-defined network slice selection procedure by means of terminal-provided NSSAI (Network Slice Selection Assistance Information) can be supported as well. Further, centralized control using SDM-C and Control Applications is optional and can be realized in an implementation-specific manner. For the 5G New Radio (NR), [10] specifies a set of key principles for slicing support which are predominantly implementation-specific and could therefore be part of dedicated control and data layer realization.

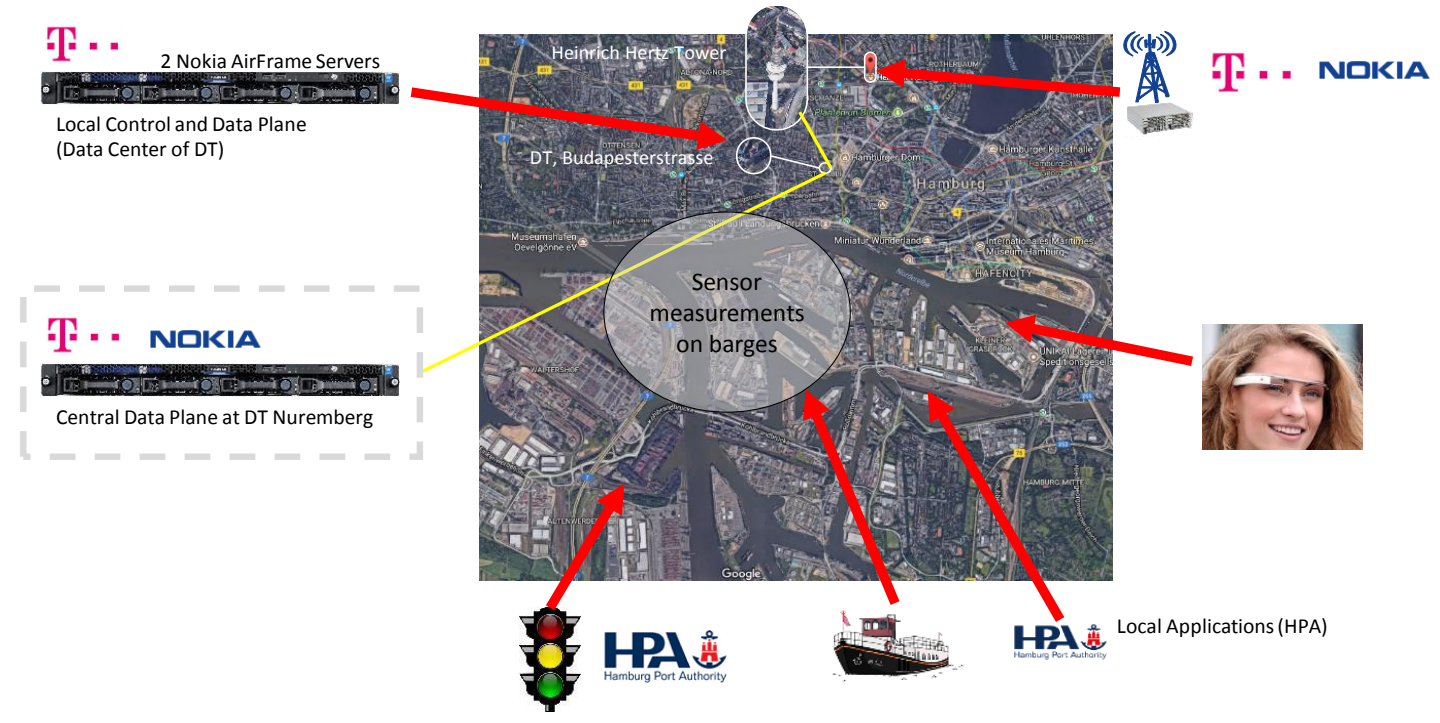

Figure 3: Physical deployment of Hamburg testbed 


\section{Logical and physical implementation}

The physical deployment of the Hamburg testbed is illustrated in Figure 3. The radio access hardware has been deployed at Heinrich Hertz Tower, Hamburg, at about 150m height using two sectorized antennas operating at $700 \mathrm{MHz}$ and using Nokia AirScale base stations. The radio equipment is connected to the DT data center Hamburg where two Nokia AirFrame servers are deployed as well as to the DT data center Nuremberg where additional virtual machines are deployed. Network functions can be executed at different cloud locations in the network to optimize application performance or resource utilization. In the trial, the (geographical) distance between the edge-cloud and the central-cloud data center was chosen large to demonstrate the benefits of an edge-cloud deployment over a fully centralized network deployment.

Furthermore, HPA provides access to a traffic light and a small number of mobile barges, which are moving within the seaport area. These devices will be connected to an application running at the premises of HPA. The devices will be either based on commercial-off-the-shelve (COTS) mobile terminals with modified software (modified 3GPP LTE Evolved Packet Core (EPC)) or software defined radio (SDR) based user equipment in the case that a modified proprietary RAN is utilized.

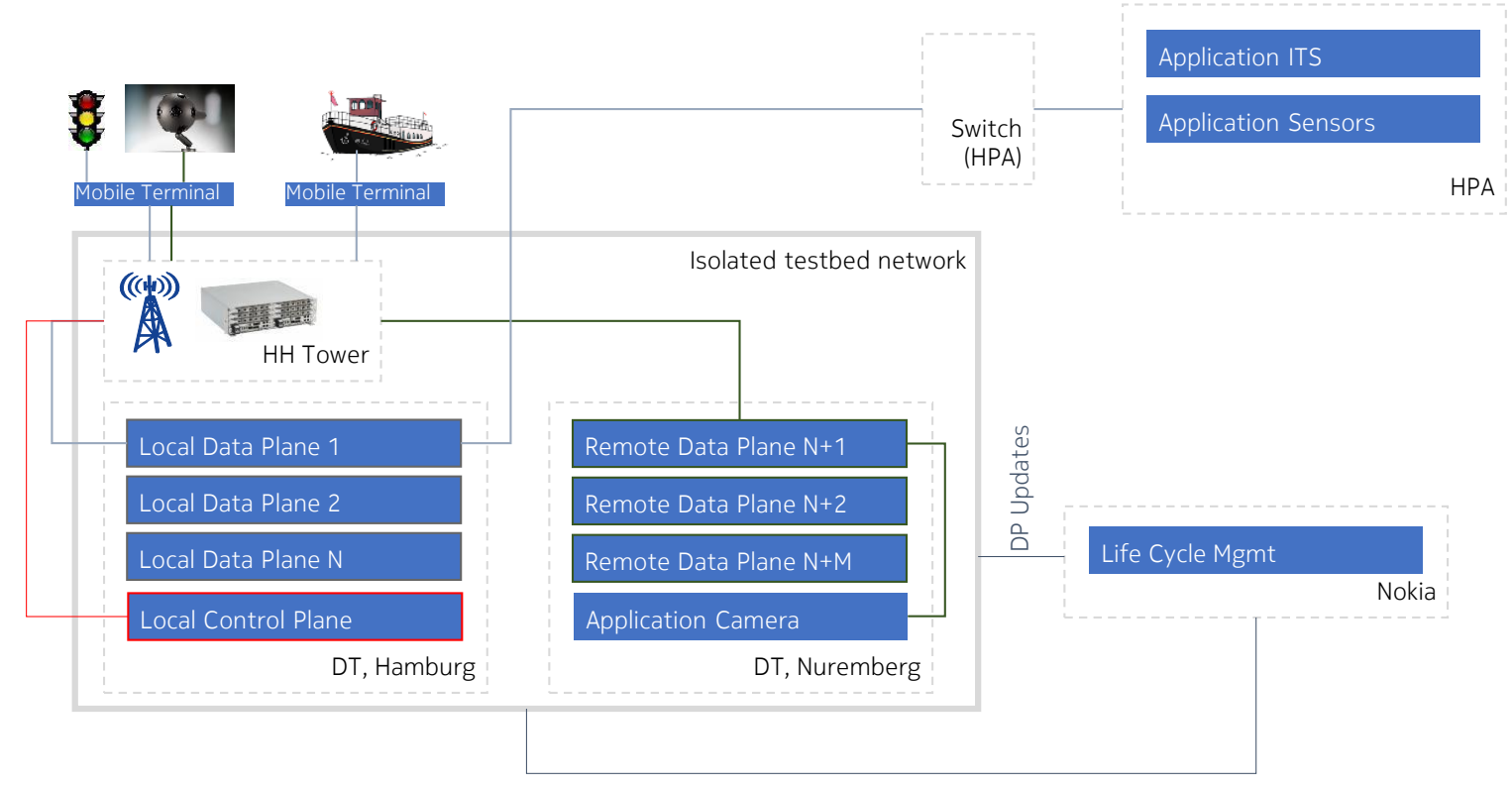

Figure 4: Logical deployment at Hamburg seaport

Based on the physical deployment, the logical setup shown in Figure 4 will be implemented. Referring to Figure 2, the testbed implements different services running in individual customized network slices, e.g., traffic light control (cMTC), environmental sensor measurement on mobile barges (mMTC), and augmented reality (eMBB). The requirements and needs of these services are defined in a Life Cycle Management (LCM) tool (see Section IV.A) where, among others, the type and location of functions can be determined or the e2e connectivity is defined (see also Section III.B). The actual setup within the LCM affects the setup of control and data plane, which are distributed and can be located at different data centers.

The network slices are set up end-to-end (e2e), i.e., both RAN and core network apply slice-specific configurations and functionality depending on the slice-type. For instance, in the case of mobile broadband services and MMTC, modified COTS user terminals are used while the RAN is slice-aware and will apply slicespecific optimizations. Each user terminal is multi-slice capable, i.e., it can connect to more than one network slice. In the case of critical MTC, SDR based terminals are used in order to provide high reliability even under mobility events (see Section IV.B). The applied security mechanisms will differ for each network slice and are tailored for the particular use case. Furthermore, the Life Cycle Management allows for configuring 
the connectivity between mobile network and applications, e.g., different virtual local area networks (VLANs) used.

\section{KEY TECHNOLOGY IMPLEMENTATIONS}

\section{A. Network Slicing and Life Cycle Management}

The lifecycle of a service or network slice comprises design/creation, instantiation/configuration, activation, run-time monitoring and re-configuration, and eventually de-activation and possibly termination as described by 3GPP [11]. Each of these phases have different demands, but taking a holistic view of the progression facilitates a smoother transition through the lifecycle and reduces overall complexity. The lifecycle is not unidirectional especially when cognitive networking and machine learning are utilized. Lessons learned later in the life of a service or slice can be fed back to earlier phases to obtain improvements in performance and quality of experience (QoE), or reduction of cost and complexity.

The perspective ideally maintained by the service and network slice lifecycle manager is an abstract view. This is the top level, e2e view of the network above network underlays, resource infrastructures, and possibly slice subnets. This abstraction allows for focusing the lifecycle manager's concern on providing a uniform platform for easily creating services, slices, and the e2e QoE assurance.

Further, high dimensional, dynamic, real-time visualizations of multiple network slices and their lifecycle transitions assist in the human understanding of the underlying physical and virtual network complexities. All of these aspects must be considered when managing services and slices throughout their lifecycles.

In the Hamburg seaport testbed, the Nokia Bell Labs E2E Service \& Network Slice Lifecyle Management tool is used to manage the services and corresponding network slices. The tool, shown in Figure 5, is a rapid development framework for research, specifically the creation and demonstration of algorithms and complex scenarios related to the E2E lifecycle management of services and network slices. It includes the offline steps of the design and creation of services and slices, as well as managing the runtime steps of orchestration, activation, and monitoring. It communicates to the underlying orchestrators and infrastructure via a set of open RESTful interfaces.



Figure 5: Illustration of Graphical User Interface of the Life Cycle Management tool

The tool is specifically used to create and manage a set of three services (mMTC, cMTC, eMBB) and their corresponding network slices. The services were created by specifying the set of service parameters that best reflect the needs of the service, e.g., mobility, throughput, latency, reliability. The network slices were created by the selection and chaining of the underlying network capabilities needed to realize the service. 
Once created, the individual network slice instances could then be configured and activated in real-time by issuing commands from the tool's 3D user interface. Once a slice is activated, the service becomes available. That is, differentiated traffic begins to flow and the over-the-top applications making use of the service can be seen to immediately begin functioning. Changes in radio resource utilization, latency, and throughput can be seen to take effect via the tool's key performance indicator (KPI) visualizations. At some subsequent point, a slice could be de-activated and terminated releasing its used resources and halting traffic flow.

As the platforms evolve, the network slice lifecycle management operations are expected to execute within a fully cognitive framework. Dynamic adaptation to the user and the network environment in small timescales will be possible by preventive, proactive actions driven by a wide range of input vectors.

\section{B. High Reliability through Multi-Connectivity}

As mentioned in Section II.C, reliability and service determinism are particularly important for the operation of the Hamburg seaport. Fulfilling low latency and reliability at the same time is challenging with existing technologies, particularly when mobility from one cell to another is involved such as on mobile barges used in the testbed. Even a successful LTE handover causes a data interruption of $\sim 40-50 \mathrm{~ms}$. Even more critical are mobility failures, which will induce interruption up to several seconds. Hence, new mobility methods have to be specified which remove the data interruption during a successful handover (" 0 ms handover" as required by the ITU-R [12]), massively improve the robustness, and remove mobility failures almost entirely.

Multi-connectivity has been identified to be a solution to the above challenge. Dual Connectivity (DC) as specified in LTE Rel-12 [6] is an inter-frequency feature. It improves small cell mobility, in case there is an umbrella macro cell on a different frequency layer acting as mobility anchor. Unfortunately, this does not solve the mentioned problems. Boundaries between macro cells are still unprotected, interruption and failures on those boundaries will remain. Furthermore, although failures between small cells can be avoided using macro-cell fallback, interruptions will still occur since packets stored in failed small cells will suffer from delay until they are retransmitted by the macro cell (or another small cell). Reordering will also stop the delivery of subsequent packets to higher layers leading to an end-to-end interruption.

The latter problem is resolved by packet duplication, i.e. by sending the same packet via multiple legs of the connection. Duplication has already been specified for the NR-packet data convergence protocol (PDCP) layer [13]. It is defined flexibly such that it allows duplication via multiple carriers when carrier aggregation is used, or eventually via multiple gNBs when dual connectivity is used. Such a duplication feature has to be handled with care, since capacity is invested (the same packet is transmitted several times).

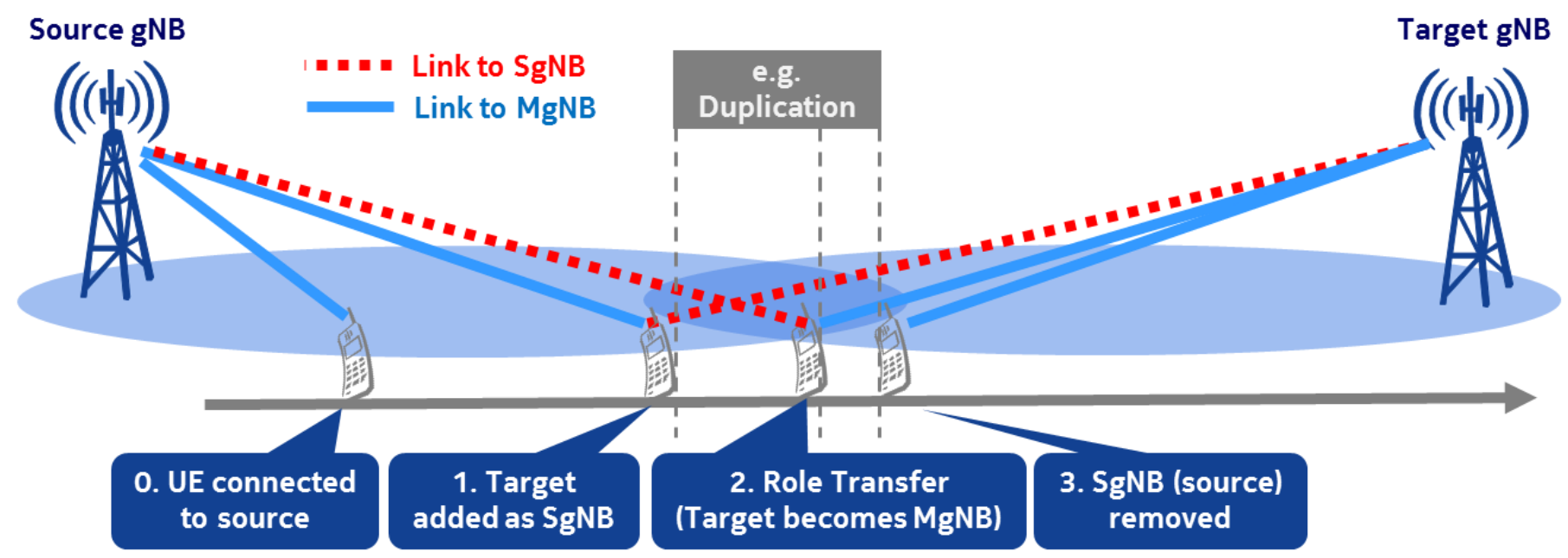

Figure 6: Implemented intra-frequency dual-connectivity approach [14] 
The former problem of unprotected macro boundaries is addressed by the DC principle applied to cells on the same frequency layer. This approach has been described in [14][15] and is implemented in this testbed (as illustrated in Figure 6). The basic idea is to set up a handover target at an earlier stage as a secondary gNB $(\mathrm{SgNB})$. Then, source (=master $\mathrm{gNB}, \mathrm{MgNB})$ and target $(=\mathrm{SgNB})$ can be simultaneously used, e.g., by duplicating packets via both nodes. At some point, the master role is transferred to the target, but the source (now SgNB) would be kept as fallback until the new connection with the target is safe. This method can remove the interruption completely, i.e., it enables a "Oms handover." Furthermore, it can improve robustness due to simultaneous usage of source and target in particular if radio bearers are duplicated.

Just as LTE DC, such a DC-based handover would require two transmitters and receivers. It may even happen that multiple target $\mathrm{SgNBs}$ need to be supported to meet extreme reliability requirements. Further concerns have been raised (e.g. interference issues, RF issues), which will be evaluated and trialed in the seaport testbed. Although 3GPP has already identified DC-based handover as the only solution for an interruptionfree and ultra-reliable handover, its standardization has been postponed to 3GPP Release 16. The implementation shown in this testbed is a proprietary SDR implementation properly modifying 3GPP LTE RAN protocols.

\section{LESSONS LEARNED AND FURTHER CHALLENGES}

\section{A. Conclusions}

The testbed proves in a real, large-scale industrial environment that basic features of network slicing, namely slice isolation, flexible slice customization and multi-tenancy, are technically feasible already today. We selected three representative services, cMTC, mMTC, and eMBB, respectively, which are all demonstrated in one testbed. Network slicing will be a key success factor for $5 \mathrm{G}$ because it allows for providing flexible, customized logical networks for different industries including automation industries or logistics. However, the testbed also showed that all network domains must be involved in the setup of network slices, i.e., user terminals, RAN, CN, and enterprise networks, in order to efficiently operate and manage network slices. Furthermore, the LCM is key for the interaction between the MSP and tenants in order to simplify and customize $5 \mathrm{G}$ network slices.

In addition, the testbed implements intra-frequency multi-connectivity for high-reliability. High-reliability is a key requirement for many IoT services such as traffic light control (cMTC) or even sensor measurements (mMTC). Most of these services not necessarily require ultra-low-latency but rather determinism and constant latencies. This is particularly difficult if devices are mobile and the channel characteristics permanently change such as in the case of barges. This testbed shows that the perspective 3GPP Release-16 feature intrafrequency multi-connectivity combined with packet duplication enables such high-reliability in order to provide the tools for diverse industrial services.

\section{B. Further Challenges and Outlook}

Beside the implementation of network slicing and multi-connectivity, the adoption of the terminals has been very challenging due to the lack of open and reliable implementations of 3GPP compliant user terminals. Furthermore, the inclusion of 5G mobile network services also poses a challenge for tenants such as HPA because current infrastructure is mostly based on wire-line networks. Integrating $5 \mathrm{G}$ mobile terminals into existing networks and processes, and the introduction of novel services exploiting the possibilities of $5 \mathrm{G}$ will be a key success factor for both telecommunication service providers and tenants, where network slicing will play key role.

A major challenge of the testbed implementation is the implementation of end-to-end network slicing, i.e., making sure that terminal, RAN, core network, and packet data network are part of a network slice and can be managed using a single entity, which is in our case the LCM. Although such an e2e setup is highly complex and involves many different domains, technologies, services, and requirements, the management and control must be very simple and intuitive in order to be applicable for tenants such as HPA. Therefore, "Zero-touch 
network and service management" (as aimed by ETSI ZSM) supported by novel machine learning algorithms is instrumental to operate such a network. Furthermore, the algorithms demonstrated in this testbed involve both terminal and network side, i.e., all changes must be applied to both sides, which poses a significant challenge for the implementation of such a testbed and the deployment of novel services. Therefore, on the one hand, flexible and reliable SDR platforms are needed for fast testbed implementations, and on the other hand, future terminals must provide more flexibility as regards interface implementations.

This testbed is implemented as part of the European project 5G-MoNArch, and its implementation permanently improves. Results will be published on the project's website (https://5g-monarch.eu/). Furthermore, first measurement results and more detailed descriptions of the implementation, deployed hardware and software, and the use cases are provided in the project report D5.1 available on the project's website. Final measurement and evaluation results will be available in the project report D5.2 in June 2019.

\section{ACKNOWLEDGEMENT}

This work has been performed in the framework of the H2020-ICT-2014-2 project 5G NORMA and the H2020-ICT-2016-2 project 5G-MoNArch. The authors would like to acknowledge the contributions of their colleagues. This information reflects the consortium's view, but the consortium is not liable for any use that may be made of any of the information contained therein.

\section{REFERENCES}

[1] Digitalization of Seaport, Prof. Jahn, Dr. Saxe, et al., 2017, Fraunhofer Verlag

[2] NGMN Alliance, NGMN $5 G$ White Paper, February 2015; https://www.ngmn.org/uploads/media/NGMN_5G_White_Paper_V1_0.pdf

[3] P. Rost, A. Banchs, I. Berberana, M. Breitbach, M. Doll, H. Droste, C. Mannweiler, M. A. Puente, K.s Samdanis, and B. Sayadi, "Mobile network architecture evolution toward 5G," IEEE Communications Magazine 54, no. 5 (2016): 84-91

[4] P. Schneider, S. Kerboeuf, and C. Mannweiler, "Providing strong 5G mobile network slice isolation for highly sensitive third-party services," Proceedings of the IEEE Wireless Communications and Networking Conference (WCNC), April 2018

[5] H2020 5G-PPP Project 5G NORMA, "Deliverable D3.3: 5G NORMA network architecture - final report,” DOI 10.5281/zenodo.1120247, October 2017

[6] 3GPP TS 36.300, "Evolved Universal Terrestrial Radio Access (E-UTRA) and Evolved Universal Terrestrial Radio Access Network (E-UTRAN); Overall description; Stage 2", V14.0.0, September 2016

[7] 3GPP TS 23.711, "Enhancements of Dedicated Core Networks selection mechanism," V14.0.0, September 2016

[8] ETSI GS NFV-MAN 001 V1.1.1, "Network Functions Virtualisation (NFV); Management and Orchestration." Dec. 2014, http://www.etsi.org/deliver/etsi_gs/NFV-

MAN/001_099/001/01.01.01_60/gs_NFV-MAN001v010101p.pdf

[9] 3GPP TS 23.501 "System Architecture for the 5G System; Stage 2", V15.0.0 (2017-12)

[10]3GPP TS 38.300 “NR and NG-RAN Overall Description; Stage 2”, V15.0.0 (2017-12)

[11]3GPP TR 28.530 "Management of network slicing in mobile networks; Concepts, use cases and requirements", V0.2.0 (2017-10)

[12]ITU-R, „Minimum requirements related to technical performance for IMT-2020 radio interface(s),“ Document ITU-R SG05 Contribution 40 
[13]3GPP TS 38.300, "NR; NR and NG-RAN Overall description; Stage 2," V15.0.0, January 2018

[14]I. Viering, H. Martikainen, A. Lobinger, B. Wegmann, "Zero-Zero Mobility: Intra-Frequency Handovers with Zero Interruption and Zero Failures," IEEE Network Magazine, March, 2018.

[15] Nokia, R2-1708588, "Dual connected intra-frequency handover for $0 \mathrm{~ms}$ interruption and mobility robustness", 3GPP TSG-RAN WG2 \#99, Berlin, Germany 


\section{BIOGRAPHIES}

Peter Rost [SM] (peter.m.rost@ nokia.com) received his Ph.D. degree from Technische Universität Dresden in 2009 and his M.Sc. degree from the University of Stuttgart in 2005. Since May 2015, he has been a member of the Radio Systems research group at Nokia Germany, contributing to the European projects 5G-NORMA and 5G-MoNArch, the German project TACNET 4.0, and business unit projects on 5G architecture. He serves as a member of VDE ITG Expert Committee on Information and System Theory, and as Executive Editor of IEEE Transactions on Wireless Communications.

Markus Breitbach (m.breitbach@telekom.de) received his Ph.D. (Dr.-Ing.) from the University of Ulm, Germany, in 1997, his diploma in electrical engineering from the Technical University of Aachen, Germany, and an MBA from the University of Applied Science Neu-Ulm, Germany, in 2006. After 7 years in the development of $3 \mathrm{G}$ base stations, he joined Deutsche Telekom in 2006, where he has been working on endto-end network architectures, in particular EPC virtualization, and contributed to EU-funded projects 5G NORMA and 5G MoNArch.

Hendrik Roreger (Hendrik.Roreger@hpa.hamburg.de) has been serving as Head of Business Intelligence at Hamburg Port Authority since end of 2017. Before he worked in the unit digital affairs and business models of HPA. Before joining HPA he has been with different IT-consulting companies for more than 5 years. He has gained experience in several ERP- and PLM-Project for various customers as project leader and process consultant. He started his career in software development after reaching a M.Sc. in Computer Science at University of Münster (Germany).

Christian Mannweiler (christian.mannweiler@nokia.com) received his M.Sc. (Dipl.-Wirtsch.-Ing.) and Ph.D. (Dr.-Ing.) degrees from the Technische Universität Kaiserslautern (Germany) in 2008 and 2014, respectively. Since 2015, he is a member of the Cognitive Network Management research group at Nokia Bell Labs, where he has been working in the area of network management automation and SON for 5G systems. $\mathrm{He}$ is (co-)author of numerous articles and papers on wireless communication technologies and architectures for future mobile networks. Christian has worked in several nationally and EU-funded projects covering the development of cellular and industrial communication systems.

Ingo Viering (viering@nomor.de) is co-founder and CEO of Nomor Research GmbH located in Munich, Germany. Furthermore, since 2007 he is also senior lecturer at Munich University of Technology. He got his Dr.-Ing. from University of Ulm in 2003, and his Dipl.-Ing. degree from the University of Technology Darmstadt in 1999. He has filed more than 100 patents, published more than 100 scientific papers, and he is actively contributing to 3GPP. In 2009, he was awarded the VDE Award for the achievements of Nomor Research.

Ray Miller (ray.miller@nokia.com) received an electrical engineering degree from Rutgers University in 1987. He has worked on advanced systems and architectures at Bell Labs for over 20 years with responsibilities and duties in a wide range of telecommunications technologies including core optical systems, metro Ethernet systems, and 3G/4G wireless systems. Currently, he is a member of the End-to-End Mobile Network Solutions Performance and Optimization research group working on the definition and optimization of $5 \mathrm{G}$ services and network architectures.

Bilgehan Erman (bilgehan.erman@ nokia-bell-labs.com) is a member of the Autonomic Networks \& Mobile Services organization within the E2E Mobile Network Solutions Laboratory at NOKIA Bell Labs. Bilgehan's recent research activities included network virtualization and 5G mobile network architectures. Throughout his career Bilgehan worked on diverse topics including cognitive data analysis and performance analysis for mobile networks, network operations, data modeling and high-dimensional visualization. He frequently contributes to projects across Bell Labs, such as the music synthesizer for Human Digital Orchestra. He holds MS degree in Computer Engineering and BS degree in Electrical Engineering both from METU at Ankara Turkey, and is an avid MOOC participant. Bilgehan has nine patents. 